\title{
Entanglement in the adiabatic limit of a two-atom Tavis-Cummings model
}

\author{
C Lazarou and B M Garraway \\ Department of Physics and Astronomy, University of Sussex, Falmer, BN1 9QH, UK \\ E-mail: c190@sussex.ac.uk
}

\begin{abstract}
We study the adiabatic limit for the sequential passage of atoms through a high-Q cavity, in the presence of frequency chirps. Despite the fact that the adiabatic approximation might be expected to fail, we were able to show that for proper choice of Stark-pulses this is not the case. Instead, a connection to the resonant limit is established, where the robust creation of entanglement is demonstrated. Recent developments in the fabrication of high-Q cavities allow fidelities for a maximally entangled state up to $97 \%$.

PACS numbers: 42.50.-p, 42.50.Pq, 03.67.Bg
\end{abstract}

Submitted to: Phys. Scr. 


\section{Introduction}

Entanglement, is a special type of correlation between two or more interacting quantum systems [1, 2]. Since they are without any classical analogy, generating such correlations has become important for the purposes of Quantum Computing and Quantum Information [3, 4]. Among the experimental demonstrations of entanglement, is that with atomic cavity QED systems in the microwave regime [5]. Apart from the generation of EPR states [6, 7], a phase gate [8, 9] has been realised along with the creation of Schrödinger cats [10].

One of the problems encountered when considering atoms traversing a cavity resonator, is that of the spatial effects due to the structure of the resonator mode [11. As long as the atoms are moving fast [12], then one can safely assume that the motion of the atomic center of mass is classical, and utilize the spatial dependence of the coupling functions with time dependent pulses [13, 14, 15. Based on this assumption, in recent works we were able to study the adiabatic sequential passage of atoms through a cavity [16, 17]. The main feature for the system is the existence of a pure crossing in the adiabatic limit, along with a number of possible applications in Quantum Computing, including atomic entanglement.

Here we study the off-resonant limit for the two-atom time-dependent TavisCummings Hamiltonian [18, 19], where now the atomic transition frequencies are subjected to time-dependent chirps. Although one would expect the adiabatic approximation to fail, the main result is that with a proper choice of Stark-shifting pulses, the system follows a similar adiabatic evolution as in the resonant limit [16]. In addition to this, the use of a chirp is a robust tool for fine tuning the adiabatic phases and consequently the output of all possible applications. When taking into account the recent developments in the engineering of high quality cavities [20, 21], we are able to demonstrate the generation of a maximally entangled state with very high fidelity.

The paper is organised as follows: In section 2 the system and the interaction Hamiltonian are introduced and after deriving the evolution matrix, we demonstrate how a maximally entangled state is formed. Section 3 gives a brief summary of the properties of entanglement with respect to the mode structure, and in section 4 we discuss the importance of spontaneous emission and decoherence. We summarize our results in section 5 ,

\section{Time-dependent Tavis-Cummings model}

The system under consideration consists of two atoms sequentially crossing an open spherical mirror cavity with velocities $v_{1}=v_{2}=v$, figure 1. The atoms enter the cavity with a time delay $2 \Delta t$, where in addition to this they can follow different trajectories inside the cavity. The Hamiltonian in the rotating wave picture and within the rotating 


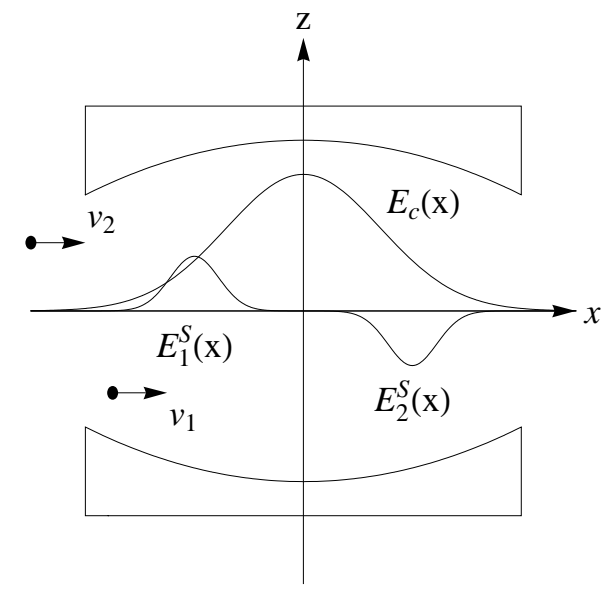

Figure 1. A pair of atoms with velocities $v_{1}=v_{2}=v$ enter a Gaussian mode cavity of width $2 w_{0}$ with a delay $2 \Delta t$. Two Gaussian EM pulses $E_{j}^{S}(x)$ are used to Stark-shift the atoms at different times. The $y$-axis is into the page.

wave approximation reads $(\hbar=1)$ [22]

$$
\hat{H}(\tau)=\sum_{j=1,2} \frac{\Delta_{j}(\tau)}{2} \hat{\sigma}_{z}^{j}+\sum_{j=1,2} \eta_{j}(\tau)\left(\hat{a}^{\dagger} \hat{\sigma}_{-}^{j}+\hat{a} \hat{\sigma}_{+}^{j}\right),
$$

where $\Delta_{j}(\tau)$ is the detuning of the $j$-th atom from the cavity mode.

For a Gaussian mode of width $2 w_{0}$ [11], the coupling functions $\eta_{j}$ have the following form

$$
\begin{aligned}
& \eta_{1}(\tau)=g_{1} \exp \left(-(\tau+\delta)^{2}\right), \\
& \eta_{2}(\tau)=g_{2} \exp \left(-(\tau-\delta)^{2}\right),
\end{aligned}
$$

where in general $g_{1} \neq g_{2}$. The dimensionless time $\tau$ and delay $\delta$ are defined in terms of the interaction time $\sigma$ to be

$$
\tau=\frac{t}{2 \sigma}, \quad \delta=\frac{\Delta t}{2 \sigma}, \quad \sigma=\frac{w_{0}}{v} .
$$

In deriving Hamiltonian (11), we are assuming that the center of mass motion is classical, and for this to be the case the atoms must be fast to avoid being reflected by the mode field [12].

For resonant interactions, i.e. $\Delta_{j}=0$, the adiabatic limit for the above Hamiltonian proved to have a rather interesting feature [16, 17]. At a finite time $\tau_{c}=\ln \left(g_{1} / g_{2}\right) /(4 \delta)$, a pure energy crossing between two of the adiabatic states is observed. This occurs in the vicinity of a temporal degeneracy and results from the absence of coupling between the two degenerate states. As a consequence of this effect, the evolution matrix has a simple form, leading to a conditional entanglement between the atoms and the cavity mode. Furthermore, in the case of equal interactions, $g_{1}=g_{2}$, a number of applications in the field of Quantum Computing such as logic gates, state mapping and teleportation, 
can be realised where the output can be tuned by means of a single dynamical phase parameter which defines the entire system evolution.

\subsection{Frequency chirps}

An interesting problem that arises when considering applications based on this system, is that of the fine-tuning of the output state. In this paper, we show how the Starkshift technique, which is used to tune the interaction time in experiments with Rydberg atoms [5], can be used to deliver a robust control over the output state.

The whole idea requires the use of smooth Gaussian frequency chirps, such that each atom experiences a detuning $\Delta_{j}(\tau)$ at different times:

$$
\begin{aligned}
& \Delta_{1}(\tau)=\Delta_{0} \exp \left(-\left(\tau+\tau_{0}\right)^{2} / \sigma_{s}^{2}\right), \\
& \Delta_{2}(\tau)=-\Delta_{0} \exp \left(-\left(\tau-\tau_{0}\right)^{2} / \sigma_{s}^{2}\right) .
\end{aligned}
$$

These atomic detunings can be produced with Gaussian EM pulses. When the first atom enters the cavity, a weak EM pulse with a spatial distribution $E_{1}^{S}(x)$, figure 1, is used to Stark-shift the atom, while crossing through the narrow region of $E_{1}^{S}(x)$. Once the first atom has crossed that region, the EM pulse is turned off, long before the second atom enters the cavity. In a similar way, a second pulse $E_{2}^{S}(x)$ is used after the first atom exits the cavity to shift the atomic transition frequency of the second atom, figure 1. If both pulses have a width $2 L$ and they have a peak at $x=-x_{0}$ and $x=x_{0}$ respectively, then the time $\tau_{0}$ and the width $\sigma_{s}$ will be

$$
\tau_{0}=\frac{v \Delta t+x_{0}}{2 w_{0}}, \quad \sigma_{s}=\frac{L}{w_{0}} .
$$

In the adiabatic limit, the system evolution will be described by the time-dependent eigenfunctions of the Hamiltonian (1). Although the analytic expressions for the adiabatic states can be derived, the whole process is lengthy [16] and beyond the scope of this paper. Here we will demonstrate the basic features of the system by means of numerical simulations with the Schrödinger equation and along with simple qualitative arguments explain the main features of the system.

Assuming that the Stark-chirps have a short duration, $\sigma_{s} \ll 1 \quad\left(L \ll w_{0}\right)$, and that the location of them, $\tau= \pm \tau_{0}$, is away from the crossing point $\tau_{c}$ for the resonant limit, the propagator for symmetric interactions, $g_{1} \approx g_{2}=g_{0}$, will have a similar form as in the resonant limit [16], i.e.

$$
\begin{aligned}
& \left|n ; e_{1}, e_{2}\right\rangle \rightarrow\left|n ; e_{1}, e_{2}\right\rangle, \\
& \left|n+1 ; g_{1}, e_{2}\right\rangle \rightarrow-\left|n+1 ; e_{1}, g_{2}\right\rangle, \\
& \left|n+1 ; e_{1}, g_{2}\right\rangle \rightarrow \cos \left(\tilde{\phi}_{n}\right)\left|n+1 ; g_{1}, e_{2}\right\rangle-\mathrm{i} \sin \left(\tilde{\phi}_{n}\right)\left|n+2 ; g_{1}, g_{2}\right\rangle, \\
& \left|n+2 ; g_{1}, g_{2}\right\rangle \rightarrow-\mathrm{i} \sin \left(\tilde{\phi}_{n}\right)\left|n+1 ; g_{1}, e_{2}\right\rangle+\cos \left(\tilde{\phi}_{n}\right)\left|n+2 ; g_{1}, g_{2}\right\rangle,
\end{aligned}
$$


where in terms of the resonant dynamical phase $\phi_{n}$, the corresponding off-resonant adiabatic phase $\tilde{\phi}_{n}$ reads

$$
\tilde{\phi}_{n}=\phi_{n}+2 \sigma \int_{-\infty}^{\infty} \mathrm{d} \tau\left(\sqrt{\Delta_{1}(\tau)^{2}+4(n+2) \eta_{1}^{2}(\tau)}-2 \eta_{1}(\tau) \sqrt{n+2}\right) .
$$

The dynamical phase $\phi_{n}$, is the integral over all time for the time-dependent energy of one of the adiabatic states in the resonant limit [16].

The second term in Equation (7) is derived after taking into account the fact that when $\Delta_{1}(\tau)$ or $\Delta_{2}(\tau)$ is on, then $\eta_{2}(\tau)=0$ or $\eta_{1}(\tau)=0$ respectively. Thus the system will correspond to a single atom interacting with the cavity mode, with a time-dependent detuning between the atomic transition and the mode frequency. Because of the choice made for the chirp, the adiabatic energies at this stage of the evolution will be pushed away from each other, and the system will adiabatically evolve during the time interval for which the atoms go through the fields $E_{j}^{S}(x)$.

When the chirp is off, the atom has crossed the region of the field $E_{j}^{S}(x)$, the system returns to the initial two-atom resonant adiabatic state, with a phase shift emerging from the chirped interaction of each atom with the mode. Since $\Delta_{1}(\tau)=-\Delta_{2}(-\tau)$ and $\eta_{1}(\tau)=\eta_{2}(-\tau)$ the two contributions, one for each atom, can be merged into one term as in Equation (7). Between the two chirps the system evolves as in the resonant limit, resulting in an additional phase $\phi_{n}$, first term in Equation (7).

Thus in the adiabatic limit and for symmetric interactions, the system evolves according to $(6 a d)-(6 d)$. For this to be the case the previously derived conditions for using the adiabatic approximation must be satisfied [16]. More specifically the coupling between the atoms and the cavity mode must be strong, $g_{0} \gg v / w_{0}$, and the delay time between the atoms must be of the order of $4 w_{0} / v$. In addition to these conditions, the EM fields used to produce the Stark-chirps must be far from the cavity center, $x_{0} \gg w_{0}$, and their width must be smaller than the mode width, $L \ll w_{0}$. It is also important to note that the pulses should be weaker than the atom-cavity coupling i.e. $\Delta_{0} \ll g_{0}$. As we see later on, violation of this condition is not detrimental for the system.

\subsection{Maximally entangled atoms}

Equations ( $(6 a)$ to $(\underline{6 d})$ describe the conditional entanglement of the second atom with the cavity mode. In order for this to be the case, the atom must be initially in its ground state. Instead of entangling the cavity mode with one of the atoms, one could generate a maximally entangled state of the two atoms. Preparing the system in the factored state

$$
\left.|\psi\rangle=\frac{1}{2}|0\rangle\left(\left|g_{1}\right\rangle+\mathrm{e}_{1}\right\rangle\right)\left(\left|g_{2}\right\rangle+\left|e_{2}\right\rangle\right)
$$

before sending the atoms through the cavity, will result in a maximally entangled state of the atoms

$$
\left|\psi_{\text {en }}\right\rangle=\frac{1}{2}\left|g_{2}\right\rangle\left(\left|g_{1}\right\rangle-\left|e_{1}\right\rangle\right)+\frac{1}{2}\left|e_{2}\right\rangle\left(\left|g_{1}\right\rangle+\left|e_{1}\right\rangle\right),
$$




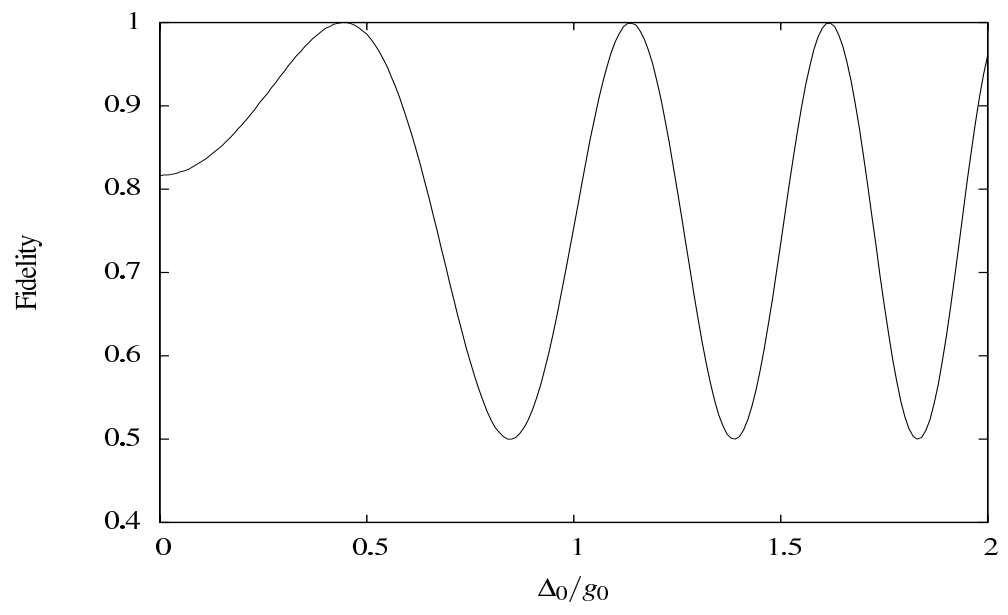

Figure 2. The fidelity for a maximally entangled state of the atoms (9) as a function of the chirp amplitude $\Delta_{0}$. The fidelity was calculated after numerical integration of the Schrödinger equation. The parameters are: $g_{0}=30 / \sigma, \delta=1.25, \tau_{0}=2.0$ and $\sigma_{s}=0.2$. The first peak where a maximally entangled state is formed is for $\Delta_{0} \approx 0.44 g_{0}$. The value obtained from Equations (7) and (10) is $\Delta_{0} \approx 0.46 g_{0}$.

if $\tilde{\phi}_{n}=2 m \pi$ with $m$ an integer. Thus, a maximally entangled state of the two atoms can be generated and the output can be fine-tuned by means of the Stark-chirps.

In figure 2] the fidelity for a maximally entangled state [3] is given for different values of the chirp amplitude $\Delta_{0}$. The first important feature is the periodic reappearance of a maximally entangled state even for $\Delta_{0} \geq g_{0}$. This is due to the fact that the system still evolves adiabatically, even if the Stark fields are stronger than the atom-cavity coupling. For $\Delta_{0}<g_{0}$ the phase $\tilde{\phi}_{n} \propto \Delta_{0}^{2}$ and the corresponding error for the fidelity

$$
F\left(\Delta_{0}\right)=\frac{\left|3+\cos \left(\tilde{\phi}_{n}\right)\right|}{4},
$$

will be a linear function of $\Delta_{0}$. Thus the Stark-shift technique is expected to be robust for weak chirps $\Delta_{0} \ll g_{0}$.

The robustness is demonstrated in figure 3 where the variations for the fidelity around the optimum value $\Delta_{\max }=0.44 g_{0}$ are plotted as a function of the ratio $\Delta_{0} / \Delta_{\max }$. From this we can see that variations of the order of $10 \%$ around the optimum value $\Delta_{0}=\Delta_{\max }$ have a very small impact on the fidelity, $(<1 \%)$. Thus generating a maximally entangled state, or realising the previously proposed applications [16, 17], can be robust by means of weak EM fields used to detune the atomic transition frequencies from the cavity frequency. This in return will induce a second order shift in the dynamical phases (77), which can be used to fine-tune the output of the evolution under the Hamiltonian (1) in the adiabatic limit. 


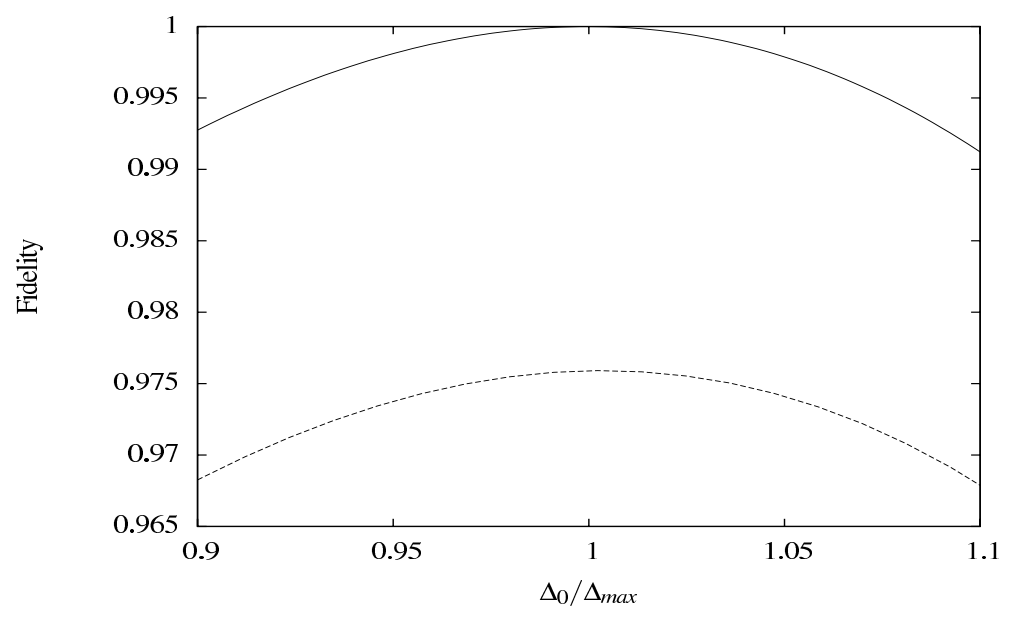

Figure 3. The fidelity for a maximally entangled state (solid) for variations of $\Delta_{0}$ around $\Delta_{\max }=0.44 g_{0}$. The fidelity (dashed) for a high quality cavity, $Q=4.2 \times 10^{10}$ [20, and circular Rydberg atoms with lifetimes $T_{a t}=30 \mathrm{~ms}$ [5]. Other parameters: $g_{0}=30 / \sigma, \delta=1.25, \tau_{0}=2.0$ and $\sigma_{s}=0.2$.

\section{Entanglement spatial properties}

Up to this point, one of the main assumptions was that both atoms are coupled to the cavity field via time-dependent coupling functions with equal amplitudes $g_{1}=g_{2}$. In the most general case and for a spherical mirror resonator [11, these amplitudes will be functions of the coordinates $y_{j}$ and $z_{j}$, figure 1, of the form

$$
g_{j}\left(y_{j}, z_{j}\right)=g_{0} \cos \left(k z_{j}\right) \exp \left(-y_{j}^{2} /\left(2 w_{0}\right)^{2}\right),
$$

where $k=2 \pi / \lambda$ is the mode wavenumber. From this we see that $g_{1} \approx g_{2}$ if $z_{1} \approx z_{2}$ and $y_{1} \approx y_{2}$. What is now interesting, is the properties of entanglement when this latter condition is not satisfied.

For the resonant limit $\Delta_{j}=0$, asymmetries in the coupling profiles (2) would introduce a second adiabatic phase $\theta_{n}$, [17], in addition to $\phi_{n}$. Despite this the conditional entanglement between the second atom and the mode still remains, but now the output state could have two different forms each defined by one of the two angles $\theta_{n}$ or $\phi_{n}$. This qualitative picture holds even when the chirps (4) are taken into account. An important feature, is that $\theta_{n}$ unlike $\phi_{n}$, does not experience any shift as $\phi_{n}$ does, see Equation (7).

It is now interesting to consider the entanglement properties for the two atoms, as their positions $z_{j}$ are varied. In order to take into account the possibility of forming a tripartite entangled state of the atoms with the cavity mode, we quantify entanglement in terms of the multipartite pure state concurrence [23], which for our case is

$$
C_{3}(|\psi\rangle)=\sqrt{3-\operatorname{tr} \rho_{1}^{2}-\operatorname{tr} \rho_{2}^{2}-\operatorname{tr} \rho_{c}^{2}},
$$

where $\rho_{j}$ is the reduced density matrix for atom $j$ and $\rho_{c}$ is the reduced density matrix for the cavity mode. Furthermore, we assume that the first atom moves along an anti- 


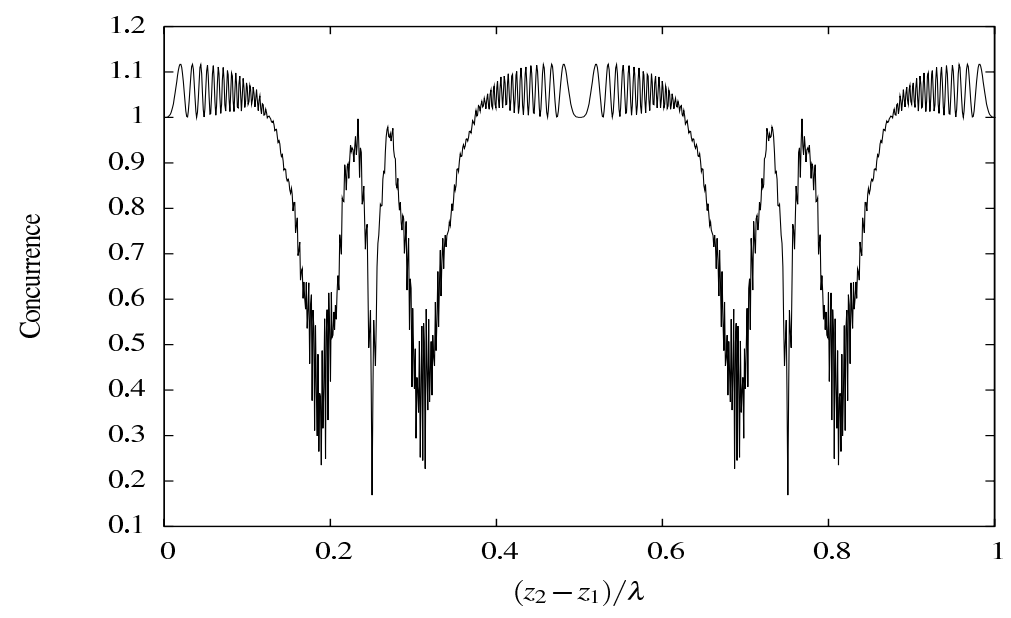

Figure 4. The concurrence (12) as a function of the atomic separation $z_{2}-z_{1}$. The initial state is $|\psi\rangle($ (8) $)$. The parameters are: $g_{0}=30 / \sigma, \delta=1.25, \Delta_{0}=0.44 g_{0}, \tau_{0}=2$ and $\sigma_{s}=0.2$.

node of the standing wave, i.e. $z_{1}=m \lambda$, while for the second atom $z_{2}$ is arbitrary. With these conditions the coupling strengths will be

$$
g_{1}=g_{0}, \quad g_{2}=g_{0} \cos \left(k\left(z_{2}-z_{1}\right)\right) .
$$

In figure 4, we plot the results of a numerical simulation for the concurrence (12), as a function of the atomic separation $z_{2}-z_{1}$. As one expects for integer or half integer values of the ratio $\left(z_{2}-z_{1}\right) / \lambda$ we have that $g_{1}= \pm g_{2}$, and a maximally entangled state of the two atoms is formed. These points are the local minima where $C_{3}(|\psi\rangle)=1$. On the other hand, whenever the atomic separation is $\left(z_{2}-z_{1}\right) / \lambda=(2 m+1) / 4$, then $g_{2}=0$ and this corresponds to the single atom Jaynes-Cummings model. For this limit, the first atom entangles with the cavity mode, forming sharp dips in figure 4 where the concurrence obtains its minimum value. Around the local minima where a maximally entangled state of the atoms is formed, symmetric spikes with $C_{3}(|\psi\rangle)>1$ signify the tripartite entanglement of the atoms and the cavity mode. For values of $C_{3}(|\psi\rangle)<1$ and arbitrary atomic separations, the system could be in either a tripartite or bipartite entangled state. In order to distinguish between the two, additional information is necessary such as the values of $\operatorname{tr} \rho_{j}^{2}$ and $\operatorname{tr} \rho_{c}^{2}$ or the populations and phases for the bare states.

\section{Spontaneous emission and cavity losses}

Spontaneous emission from the atoms to modes other than the cavity mode, and photon losses through the cavity mirrors are both detrimental for entanglement. In an attempt to quantify the importance of both effects, we solved the master equation for the density matrix of the entire system,

$$
\frac{\mathrm{d} \rho}{\mathrm{d} t}=-\mathrm{i}[\hat{H}(t), \rho]+\mathcal{L}_{s}(\rho)+\mathcal{L}_{c}(\rho) .
$$


The Liouvillian terms $\mathcal{L}_{s}(\rho)$ and $\mathcal{L}_{c}(\rho)$, respectively describe the atomic spontaneous emission with a rate $\Gamma$, and the decay of the cavity with a rate $\gamma$ into different thermal reservoirs at zero temperature [24],

$$
\begin{aligned}
& \mathcal{L}_{s}(\rho)=-\frac{\Gamma}{2} \sum_{j=1,2}\left(\rho \hat{\sigma}_{+}^{j} \hat{\sigma}_{-}^{j}+\hat{\sigma}_{+}^{j} \hat{\sigma}_{-}^{j} \rho-2 \hat{\sigma}_{-}^{j} \rho \hat{\sigma}_{+}^{j}\right), \\
& \mathcal{L}_{c}(\rho)=-\frac{\gamma}{2}\left(\hat{a}^{\dagger} \hat{a} \rho+\rho \hat{a}^{\dagger} \hat{a}-2 \hat{a} \rho \hat{a}^{\dagger}\right) .
\end{aligned}
$$

Calculating multipartite concurrence for a mixed state is not an easy task [25]. One would have to perform an optimization for the concurrence $C_{3}\left(\left|\psi_{j}\right\rangle\right)$ (12) over all pure state ensembles $\left\{\left|\psi_{j}\right\rangle\right\}$, which equally represent the mixed state $\rho$ that corresponds to the solution of Equation (14). Instead of this approach, we were able to show after a number of simulations, that the final state of the system has a simple form

$$
\rho(\infty) \approx|0\rangle\langle 0| \otimes \rho_{a}(\infty),
$$

where $\rho_{a}(\infty)$ is the reduced density matrix for the atoms for $t=\infty$. This result is for the initial state (8) , and is rather accurate since the average photon number $\langle n(\infty)\rangle$ is very small, $\langle n(\infty)\rangle<10^{-3}$, whereas the correlations between the mode and the atoms are negligible. This result, allows the calculation of the concurrence for the atomic pair, by means of the two-qubit mixed state concurrence [26, 27]

$$
c(\rho)=\max \left\{0, \sqrt{\lambda}_{1}-\sqrt{\lambda}_{2}-\sqrt{\lambda}_{3}-\sqrt{\lambda}_{4}\right\},
$$

where $\lambda_{j}$ are the eigenvalues of the matrix

$$
R(\rho)=\rho\left(\sigma_{y} \otimes \sigma_{y}\right) \rho^{*}\left(\sigma_{y} \otimes \sigma_{y}\right),
$$

where $\sigma_{y}$ is the Pauli matrix [28] and the eigenvalues $\lambda_{i}$ are in increasing order i.e. $\lambda_{1}>\lambda_{2}>\lambda_{3}>\lambda_{4}$.

The results from numerical simulations with Equation (14) and (17) are plotted in figure 5, where we see that entanglement between the two atoms exponentially decays with respect to both decay rates. Furthermore, the most important detrimental effect is that of spontaneous emission from the atoms. This is because the effective decay rate for the subsystem of the two atoms is greater than the single atom decay rate $\Gamma$. That is, entanglement is more sensitive to decay due to spontaneous emission than decay due to cavity losses.

\subsection{Experimental feasibility}

For experiments with circular Rydberg states of $\mathrm{Rb}^{85}$ atoms, with lifetimes $T_{a t}=30 \mathrm{~ms}$ [5, 21], the main requirement for adiabatic evolution is for the system to be in the strong coupling regime, $g_{0} w_{0} / v \approx 20-30$. For a coupling strength $g_{0} / 2 \pi=50 \mathrm{kHz}$ and a mode waist $2 w_{0}=6 \mathrm{~mm}$, the atomic velocities must be $v \approx 60-95 \mathrm{~m} / \mathrm{s}$. These speeds are large enough to avoid reflection of the atoms from the cavity field. In addition to this the initial atomic displacement must be $2 v \Delta t \approx(4-5) w_{0}=(12-15) \mathrm{mm}$. 

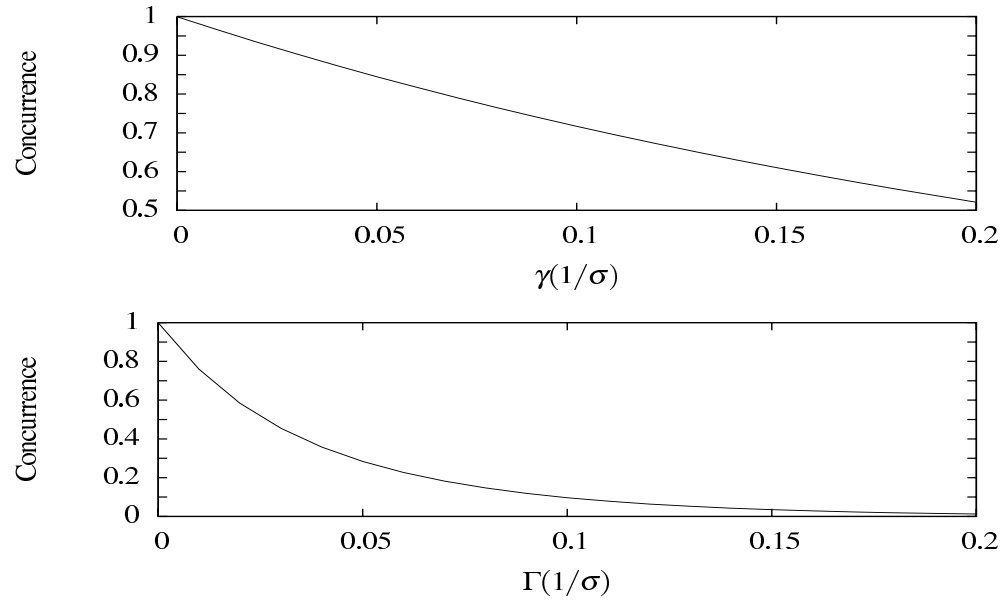

Figure 5. The two-qubit concurrence (17), for different cavity decay rates $\gamma$ with $\Gamma=0$ (top) and for different spontaneous emission rates $\Gamma$ with $\gamma=0$ (bottom). Other parameters: $g_{0} \approx 18.9286 / \sigma, \delta=1.25$ and $\Delta_{0}=0$. The initial state is (8), whereas for this parameters and for $\gamma=\Gamma=0$ the output is a maximally entangled state (9).

This distance is bigger than the width of the Stark fields which is $2 L \approx 0.4 w_{0}=$ $1.2 \mathrm{~mm}$. This allows the Stark fields to be turned on and off at proper times so that they interact with only one atom and consequently produce the desired chirps (4). The location of the Stark pulses can vary between 4.5 and $6 \mathrm{~mm}$ with no impact on the robustness of the system. In addition to all these, a recent development was the engineering of a cavity with $Q=4.2 \times 10^{10}[20,21]$. For this quality factor and the corresponding lifetimes for the circular Rydberg atoms, the fidelity for a maximally entangled state (9) is reduced by about 3\% varying between 96 and $97 \%$, figure 3, which is a very high fidelity.

\section{Conclusion}

In this work we have studied a time-dependent, off-resonant two-atom Tavis-Cummings Hamiltonian. Considering pairs of slowly moving atoms, sequentially crossing a Gaussian mode resonator, we examine the adiabatic limit for the system. As we were able to show, for proper choice of atomic frequency chirps the evolution of the system bears strong similarities to the resonant limit. The system propagator has the same structure as in the resonant limit, and one can realise the exact same applications [16, 17].

The only and also crucial difference from the resonant limit, is that the adiabatic phase is now shifted due to frequency chirps. Because of the linear dependence of this shift with respect to the chirps amplitude, all the proposed applications can be robust. As an example, the creation of a maximally entangled state of the atoms can be achieved with fidelities higher than 99\%. Despite potential detrimental effects, such as atomic 
spontaneous emission or photon losses from the resonator mirrors, recent developments in the fabrication of high quality cavities [20, 21, allow fidelities as high as 97\%. In addition to this, the adiabatic condition can be easily satisfied for experiments with Rydberg atoms in the microwave regime [5].

Although time-dependent frequency chirps could be detrimental for the adiabatic approximation, our results show that proper timing of these chirps could enable adiabatic evolution even if they are stronger than the coupling between the atoms and the field. One could again coherently tune the output of the evolution, but this would be less robust. This is due to the sinusoidal dependence of the error with respect to the chirp amplitude for strong Stark-fields. On the other hand, for weak chirps the error in the fidelity is very small making the Stark-shift technique highly robust.

\section{References}

[1] Einstein A, Podolsky B and Rosen N 1935 Phys. Rev. 47 777-80

[2] Bell J S 1964 Physics 1195

[3] Nielsen M A and Chuang I L 2000 Quantum computation and quantum information (Cambridge: Cambridge University Press) p 409

[4] Galindo A and Martín-Delgado M A 2002 Rev. Mod. Phys. 74 347-423

[5] Raimond J M, Brune M and Haroche S 2001 Rev. Mod. Phys. 73 565-82

[6] Hagley E, Maître X, Nogues G, Wunderlich C, Brune M, Raimond J M and Haroche S 1997 Phys. Rev. Lett. 79 1-5

[7] Osnaghi S, Bertet P, Auffeves A, Maioli P, Brune M, Raimond J M and Haroche S 2001 Phys. Rev. Lett. 87037902

[8] Nogues G, Rauschenbeutel A, Osnaghi S, Brune M, Raimond J M and Haroche S. 1999 Nature $400239-42$

[9] Rauschenbeutel A, Nogues G, Osnaghi S, Bertet P, Brune M, Raimond J M and Haroche S 1999 Phys. Rev. Lett. 83 5166-69

[10] Brune M, Hagley E, Dreyer J, Maître X, Maali A, Wunderlich C, Raimond J M and Haroche S 1996 Phys. Rev. Lett. 77 4887-90

[11] Yariv A 1989 Quantum Electronics (New York: John Wiley) p 136

[12] Meyer G M, Scully M O and Walther H 1997 Phys. Rev. A 56 4142-52

[13] Schlicher R R 1989 Opt. Comm. 70 97-102

[14] Marr C, Beige A and Rempe G 2003 Phys. Rev. A 68033817

[15] Yong L, Bruder C and Sun C P 2007 Phys. Rev. A 75032302

[16] Lazarou C and Garraway B M 2008 Phys. Rev. A 77023818

[17] Lazarou C and Garraway B M 2008 Eur. Phys. J. ST 160 235-46

[18] Tavis M and Cummings F W 1968 Phys. Rev. 170 379-84

[19] Tavis M and Cummings F W 1969 Phys. Rev. 188 692-95

[20] Kuhr S, Gleyzes S, Guerlin C, Bernu J, Hoff U B, Deleglise S, Osnaghi S, Brune M, Raimond J M, Haroche S, Jacques E, Bosland P and Visentin B 2007 Appl. Phys. Lett. 90164101

[21] Guerlin C, Bernu J, Deleglise S, Sayrin C, Gleyzes S, Kuhr S, Brune B, Raimond J M and Haroche S 2007 Nature 448889

[22] Shore B W 1990 The Theory of Coherent Atomic Excitation vol 2 (New York: John Wiley) p 866

[23] Mintert F, Carvalho A A R, Kus M and Buchleitner A 2005 Phys. Rep. 415 207-59

[24] Breuer H P and Petruccione F 2002 The theory of open quantum systems (New York: Oxford University Press) p 109

[25] Carvalho A R R, Mintert F and Buchleitner A 2004 Phys. Rev. Lett. 93230501 
[26] Hill S and Wootters W K 1997 Phys. Rev. Lett. 78 5022-25

[27] Wootters W K 1998 Phys. Rev. Lett. 80 2245-48

[28] Merzbacher E 1998 Quantum Mechanics (New York: John Wiley) p 386 